\title{
Chlorophyll a fluorescence illuminates a path connecting plant molecular biology to Earth-system science
}

\section{Porcar-Castell, Albert}

2021-08-09

Porcar-Castell , A , Malenovsky , Z, Magney , T , Van Wittenberghe , S , Fernandez-Marin , B , Maignan , F , Zhang , Y, Maseyk , K, Atherton , J , Albert , L P , Robson , T M , Zhao , F , Garcia-Plazaola , J-I, Ensminger , I, Rajewicz , P A, Grebe , S , Tikkanen, M , Kellner , J R, Ihalainen , J A , Rascher , U \& Logan , B 2021 , ' Chlorophyll a fluorescence illuminates a path connecting plant molecular biology to Earth-system science ' , Nature plants , vol. 7 , pÿno. 8 , pp. 9981009 . https://doi.org/10.1038/s41477-021-00980-4

http://hdl.handle.net/10138/339926

https://doi.org/10.1038/s41477-021-00980-4

unspecified

acceptedVersion

Downloaded from Helda, University of Helsinki institutional repository.

This is an electronic reprint of the original article.

This reprint may differ from the original in pagination and typographic detail.

Please cite the original version. 
1 Chlorophyll-a fluorescence illuminates a path connecting plant molecular biology to Earthsystem science

Albert Porcar-Castell ${ }^{1 *}$, Zbyněk Malenovský ${ }^{2}$, Troy Magney ${ }^{3}$, Shari Van Wittenberghe ${ }^{1,4}$, Beatriz Fernández-Marín ${ }^{5}$, Fabienne Maignan ${ }^{6}$, Yongguang Zhang ${ }^{7}$, Kadmiel Maseyk $^{8}$, Jon Atherton ${ }^{1}$, Loren P. Albert ${ }^{9}, 10$, Thomas Matthew Robson ${ }^{11}$, Feng Zhao ${ }^{12}$, Jose-Ignacio Garcia-Plazaola ${ }^{13}$, Ingo Ensminger ${ }^{14}$, Paulina A. Rajewicz ${ }^{1}$, Steffen Grebe ${ }^{15}$, Mikko Tikkanen ${ }^{15}$, James R. Kellner ${ }^{9}$, ${ }^{16}$, Janne A. Ihalainen ${ }^{17}$, Uwe Rascher ${ }^{18}$, Barry $\operatorname{Logan}^{19}$

${ }^{1}$ Optics of Photosynthesis Laboratory, Institute for Atmospheric and Earth System Research/Forest Sciences, Viikki Plant Science Center, University of Helsinki, Helsinki, Finland.

${ }^{2}$ School of Geography, Planning, and Spatial Sciences, College of Sciences Engineering and Technology, University of Tasmania, Private Bag 76, Hobart, TAS 7001, Australia.

${ }^{3}$ Department of Plant Sciences, University of California, Davis. Davis, CA, 95616 United States of America.

${ }^{4}$ Laboratory of Earth Observation, University of Valencia, C/Catedrático José Beltrán, 2, 46980 Paterna, Spain.

${ }^{5}$ Department of Botany, Ecology and Plant Physiology, University of La Laguna (ULL), Tenerife 38200, Spain.

${ }^{6}$ Laboratoire des Sciences du Climat et de l'Environnement, LSCE/IPSL, CEA-CNRS-UVSQ, Université Paris-Saclay, Gif-sur-Yvette, France.

${ }^{7}$ International Institute for Earth System Sciences, Nanjing University, Nanjing, Jiangsu 210023, China.

${ }^{8}$ School of Environment, Earth and Ecosystem Sciences, The Open University, Milton Keynes MK7 6AA, United Kingdom. 

9 0

${ }^{9}$ Institute at Brown for Environment and Society, Brown University, Providence, RI 02912, United States of America.

${ }^{10}$ Biology Department, West Virginia University, Morgantown, WV 26506-6300, United States of America.

${ }^{11}$ Organismal and Evolutionary Biology, Viikki Plant Science Centre (ViPS), Faculty of Biological and Environmental Science, 00014, University of Helsinki, Finland.

${ }^{12}$ School of Instrumentation Science and Opto-Electronics Engineering, Beihang University, Beijing, 100083, China.

${ }^{13}$ Department of Plant Biology and Ecology, University of the Basque Country (UPV/EHU), Bilbao, Spain.

${ }^{14}$ Department of Biology, Graduate Programs in Cell \& Systems Biology and Ecology \& Evolutionary Biology, University of Toronto, 3359 Mississauga Road, Mississauga, ON L5L 1C6, Canada.

${ }^{15}$ Molecular Plant Biology, University of Turku, FI-20520 Turku, Finland.

${ }^{16}$ Department of Ecology and Evolutionary Biology, Brown University, Providence RI 02912, United States of America.

${ }^{17}$ Nanoscience Center, Department of Biological and Environmental Science, University of Jyväskylä, Jyväskylä 40014, Finland.

${ }^{18}$ Institute of Bio- and Geosciences, Plant Sciences (IBG-2), Forschungszentrum Jülich GmbH, Jülich, Germany.

${ }^{19}$ Biology Department, Bowdoin College, Brunswick, Maine, United States of America.

*Corresponding author: joan.porcar@helsinki.fi 
For decades, the dynamic nature of chlorophyll-a fluorescence (ChlaF) has provided insight into the biophysics and ecophysiology of the light reactions of photosynthesis from the subcellular to leaf scales. Recent advances in remote sensing methods now enable detection of ChlaF induced by sunlight across a range of larger scales, using instruments mounted on towers above plant canopies to Earth-orbiting satellites. This signal is referred to as solarinduced fluorescence (SIF) and its application promises to overcome spatial constraints on studies of photosynthesis, opening new research directions and opportunities in ecology, ecophysiology, biogeochemistry, agriculture and forestry. However, to unleash the full potential of SIF, intensive cross-disciplinary work is required to harmonize these new advances with the rich history of biophysical and ecophysiological studies of ChlaF, fostering the development of next-generation plant physiological and Earth system models. Here, we introduce the scale-dependent link between SIF and photosynthesis, with an emphasis on seven remaining scientific challenges, and present a roadmap to facilitate future collaborative research towards new SIF applications.

When illuminated, chlorophyll-a molecules weakly emit light in the $650-850 \mathrm{~nm}$ range; that is, they fluoresce. Steady state ${ }^{1,2}$ and time-resolved fluorescence spectroscopy ${ }^{3,4}$, as well as pulse-amplitude modulated (PAM) fluorescence ${ }^{5,6}$ have long been used by biophysicists, molecular biologists and ecophysiologists to elucidate the structure and function of the photosynthetic apparatus ${ }^{7-9}$. These techniques are regarded as active because the measured ChlaF originates from a controlled light source, and accordingly have largely ${ }^{10,11}$ been restricted to measurements at the subcellular and leaf levels.

Interest in passive remote sensing methods capable of retrieving solar-induced ChlaF across a continuum of spatial scales emerged more than two decades ago ${ }^{12}$. These seminal activities led to the first demonstrations of tower-based ${ }^{13,14}$ and satellite ${ }^{15}$ SIF measurements over terrestrial ecosystems. The opportunity to remotely detect an energy flux (Box 1) that arises directly from 
within the photosynthetic process spurred the rapid development of measurement techniques, retrieval protocols, and models for estimating and interpreting SIF across scales. As reviewed in Mohammed et al. ${ }^{12}$ and Aasen et al. ${ }^{16}$, SIF can now be measured from an expanding number of sensors mounted on towers ${ }^{17,18}$, drones ${ }^{19,20}$, aircraft ${ }^{21,22}$ and satellites with ever-improving spatial and temporal resolution ${ }^{23,24}$. So far, all satellite SIF retrievals have been serendipitous, relying on instruments originally designed to measure atmospheric gases. The first satellite mission designed specifically for the measurement of SIF is the ESA FLuorescence EXplorer (FLEX) mission, which is set to launch in $2024^{25}$.

SIF methods are rapidly breaking through the scale bottleneck of traditional ChlaF measurements, opening up a range of new opportunities to study photosynthesis across the continuum of spatial scales from the leaf, through plant canopies, and up to the globe. With SIF we now have the potential to illuminate the path connecting plant molecular biology to Earth-system science. However, before the full potential of multiscale SIF observations can be realized, a number of challenges must be overcome. Extracting the information embedded in the SIF signal requires a fundamental understanding and a quantitative description of the processes that connect measured ChlaF with photosynthesis (Fig.1), as well as their variation across space and time (Fig. 2). In this Perspective, we present these challenges and propose a roadmap of activities to facilitate future research. Finally, we discuss key emerging SIF applications that can benefit from cross-disciplinary expertise.

Challenge 1: APAR. The common denominator between ChlaF and the photosynthetic uptake of $\mathrm{CO}_{2}$ is the flux of photosynthetically active radiation absorbed by photosynthetic pigments, or $\mathrm{APAR}_{\mathrm{g}}$ (where the g stands for green), which provides the foundation for the mechanistic connection between SIF and photosynthesis. APARg is the product of the incoming photosynthetically active radiation (PAR) and the fraction of this PAR absorbed by photosynthetic pigments (fAPARg) (Fig.1). Importantly, although the absorption of radiation by leaves and plant 
canopies can be quantified using radiometric sensors either coupled to an integrating sphere ${ }^{26}$ (e.g. leaf absorptance profile in Fig.1) or mounted above and below a plant canopy ${ }^{27}$, these measurements also include a significant and dynamic contribution from non-photosynthetic pigments and other canopy elements. While inaccuracies in the estimation of $\mathrm{APAR}_{\mathrm{g}}$ do not disrupt the relationship between SIF and photosynthesis, accurate quantification of the energy flux entering the photosynthetic process is essential for a mechanistic interpretation of SIF and remains a challenge.

\section{Challenge 2: Distribution of excitation energy between PSII and PSI and their ChlaF emissions.}

$\mathrm{APAR}_{\mathrm{g}}$ is absorbed mostly by chlorophyll-a and chlorophyll-b associated with either photosystem II (PSII) or photosystem I (PSI) reaction centres. Interestingly, while both types of chlorophyll have the capacity to fluoresce, essentially all chlorophyll fluorescence in vivo originates from chlorophyll-a due to the efficient transfer of excitation energy from chlorophyll-b to chlorophyll-a within light harvesting antennae ${ }^{28}$. Likewise, although both photosystems emit ChlaF, ChlaF from PSII typically dominates the signal, especially in the red region of the emission spectrum² (Fig.1), and exhibits greater variation in quantum yield in response to photochemical and nonphotochemical processes ${ }^{7,29}$. The dynamic nature of PSII ChlaF explains the widespread application of PAM fluorescence to probe the energy partitioning between photochemical and nonphotochemical processes or to estimate the rate of linear electron transport (LET) in PSII ${ }^{30}$. However, the estimation of LET requires knowledge of the distribution of absorption between the photosystems (i.e. the use of an energy partitioning factor), which is rarely measured and often assumed to be $0.5^{6}$. Although biochemical and biophysical methods to assess the stoichiometry and antenna sizes of PSI and PSII do exist ${ }^{31-33}$, these methods only provide a relative assessment of the energy distribution; absolute quantification requires the combination of simultaneous ChlaF and $820 \mathrm{~nm}$ absorption measurements to probe the energy partitioning in PSII and PSI, respectively, along with photosynthetic gas exchange measurements ${ }^{34}$. Overall, the evidence gathered to date 
suggests that neither the distribution of excitation energy between PSII and PSI nor the contribution of ChlaF from PSI to SIF remain constant over time, between species or within canopy light gradients $^{35,36}$. Questions remain: how large is this variability? What controls it? And what is its significance for the interpretation of SIF? Answers to these questions await the development of versatile field methods and protocols (e.g. based on rapid optical measurements ${ }^{37}$ ) to enable the characterization of these factors across a wide range of conditions.

Challenge 3: Energy partitioning in PSII. Energy absorbed in PSII is partitioned between three main processes: a) photochemical quenching (PQ) of excitation energy, promoting linear electron transport, b) non-photochemical quenching (NPQ), which includes both regulated and sustained forms of thermal dissipation, and c) emission of ChlaF. The quantum yield of a process, e.g. ChlaF emission $(\Phi \mathrm{F})$, can be expressed as the ratio of the rate constant associated to that process relative to the sum of all rate constants. Importantly, the rate constants associated to PQ and NPQ are highly dynamic, which allows plants to regulate the flow of energy through PSII and to protect against light-induced damage ${ }^{38,39}$. During the growing season, the rate constants of PQ and NPQ vary over time-scales of seconds to minutes in response to the redox dynamics of the quinone acceptor pool and induction and relaxation of regulated thermal dissipation, respectively. Outside of the growing season, or during periods of profound environmental stress, rate constants can be affected by photoinhibition of PQ and the induction of sustained NPQ. Accordingly, changes in the quantum yield of ChlaF $(\Phi F)$ reflect the combined effect of PQ and NPQ dynamics and a quantitative connection between $\Phi \mathrm{F}$ and $\Phi \mathrm{P}$ (the quantum yield of photochemistry) cannot be established without knowledge of either PQ or $\mathrm{NPQ}^{8,40}$. PAM fluorescence uses saturating light pulses to solve the energy partitioning and estimate $\Phi \mathrm{P}$; an approach that is not feasible during SIF measurements, precluding partitioning from SIF alone.

Under certain conditions, either NPQ or PQ can dominate the relationships between $\Phi F$ and $\Phi P$, resulting in the emergence of a positive or negative relationship respectively. For example, under 
151 low light intensities - when regulatory NPQ remains inactive - the relationship between $\Phi P$ and $\Phi F$ 152 is negative under the action of PQ, which exerts opposite effects on (i.e. decouples) $\Phi \mathrm{P}$ and $\Phi F$.

153 Under high light - when PQ tends to saturate and NPQ is highly active - the relationship between $\Phi \mathrm{P}$ and $\Phi \mathrm{F}$ turns to positive under the action of NPQ, which competes for excitation with both (i.e. couples) $\Phi \mathrm{P}$ and $\Phi \mathrm{F}^{12,52,53}$. The latter case can explain the seasonal correlation between $\Phi \mathrm{P}$ and $\Phi \mathrm{F}$ observed at the leaf ${ }^{41,42}$ (Fig. 2) and canopy scales ${ }^{18}$, in response to the modulation of sustained NPQ that protects the foliage from the harmful combination of excessive light and low temperatures ${ }^{43,44}$. Despite the positive relationship between $\Phi P$ and $\Phi F$ that emerges in response to certain stress conditions, the quantitative treatment of the energy partitioning in PSII requires the use of mechanistic models and remains one of the core challenges to the interpretation of $\operatorname{SIF}^{40,45,46}$.

Challenge 4: Alternative energy sinks. Photosynthetic linear electron transport provides reducing power for a range of metabolic processes beyond $\mathrm{CO}_{2}$ assimilation via the Calvin cycle, including chlororespiration $^{47}$, photorespiration ${ }^{48}$, nitrogen, sulphur and oxygen reduction (the latter known as the Mehler reaction in the water-water cycle ${ }^{49}$ ), and the synthesis of volatile organic compounds ${ }^{50}$. Importantly, the dynamics of these 'non-assimilatory' electron sinks can affect ChlF in a manner not directly correlated with $\mathrm{CO}_{2}$ assimilation. In particular, because alternative energy sinks can have a protective function by sustaining LET under conditions when $\mathrm{CO}_{2}$ assimilation is impaired ${ }^{51}$, they could influence the capacity of SIF to detect certain plant stress responses. Therefore, it is critical to address the extent that these dynamics decouple SIF from GPP, in particular during plant stress. As with Challenge 2, answering this question will benefit from the development of versatile field methods and protocols to promote the widespread characterization of these factors across a wide range of conditions.

\section{Challenge 5: Leaf and canopy ChlaF scattering, reabsorption and measurement geometry.}

Although the lighter and darker green stripes seen on an athletic field may give the impression of different chlorophyll contents, they are an optical reflection effect created when the grass is bent in 
a particular direction during mowing. SIF measurements over plant canopies are similarly affected by the distribution of leaves, canopy architecture and measurement geometry ${ }^{27,52}$. The amount and distribution of chlorophyll within a leaf (influenced by photosystem and thylakoid structure, chloroplast distribution, and internal leaf morphology), as well as the amount and geometrical arrangement of leaves and other non-photosynthetic material within a plant canopy (influenced by branch/stem architecture) drive APARg, connecting SIF and photosynthesis at the leaf and canopy scales, respectively. Once emitted, ChlaF photons travel through the same leaf and canopy structures, where some of the ChlaF photons are reabsorbed (Fig. 1 and Fig. 2 "spectral dynamics"). As a result, spatial and temporal variations in leaf biochemistry, leaf morphology, and canopy architecture, as well as foliage illumination and viewing geometry, influence the probability of ChlaF photons contributing to a SIF measurement (known as the escape probability). These factors decouple the total emitted ChlaF from the measured SIF, and by extension from photosynthesis. Physically-based radiative transfer models, which simulate the movement of photons through leaves and plant canopies (Box 2), can be used to provide a quantitative framework to investigate and account for the impact of these factors on APAR $\mathrm{g}$ and SIF observations ${ }^{27,53}$. Although spatially explicit RTM approaches are already available (see Supplementary Video 1 and 2), advances in the parametrization of within-leaf and canopy drivers of SIF - e.g. canopy gradients in foliar morphology, pigment contents (Challenge 1) or ChlaF contribution from PSI (Challenge 2) - remain areas of active development.

Challenge 6: Atmospheric absorption and scattering. Atmospheric gases, aerosols and other particles absorb and scatter ChlaF photons traveling from a plant canopy to a remote detector. The extent of atmospheric absorption and scattering of SIF depends on the retrieval wavelength, the distance between target and sensor, and the properties of the atmosphere (Box 1). In particular, SIF retrieval methods based on the in-filling of atmospheric gas absorption bands, such as the O2-A or O2-B bands (Fig. 1), face the challenge that the gas absorption feature used for the SIF retrieval 
simultaneously attenuates the ChlaF signal as it travels towards the detector. This effect requires a correction even for short-distance measurements from canopy towers and drones ${ }^{54}$. Although an atmospheric RTM can be used to characterize and correct for these effects, its application requires site-specific measurements of atmospheric profile parameters for model input, which remains an operational challenge ${ }^{55}$.

Challenge 7: Integrating SIF controls across space and time. A final challenge, and perhaps the most relevant, is the contextualization of the interpretation of SIF (including the previous six challenges) within the spatial and temporal domain of the measurements (Fig. 2). Temporally, ChlaF dynamics have been used to investigate the energy transfer within photosystems (femtopicosecond scale $)^{7,56}$, the redox status of the donor and acceptor sides of the photosystem (microsecond-millisecond scale) $)^{3,4}$, and the variations in PQ and NPQ (seconds-to-seasonal scale) ${ }^{38}$, ${ }^{39,44}$. Spatially, the intensity and spectral properties of SIF are also controlled by factors that regulate both $\mathrm{APAR}_{\mathrm{g}}$ and ChlaF scattering and reabsorption within a leaf or plant canopy ${ }^{57,58}$ (Fig. 2, "spectral dynamics"). When ChlaF is measured as SIF across coarser spatial and longer temporal scales, the signal carries information that aggregates an expanding assortment of physical and biological factors ${ }^{59-61}$. New controls may appear while the effects of others may be subordinated, strengthening (via 'couplers'; Fig. 2) or disrupting (via 'decouplers'; Fig. 2) the relationship between SIF and GPP.

For example, tower-based SIF studies reveal a strong seasonal linear relationship between canopy SIF and ecosystem GPP across a wide range of ecosystems ${ }^{17,18,61}$, consistent with the coupling action of APARg and NPQ described above. Yet, the sensitivity, strength and linearity of the seasonal SIF-GPP relationship is not universal and has been found to depend on additional physical and physiological decoupling factors, such as sun-vegetation-sensor geometries ${ }^{62,63}$, vegetation canopy structure ${ }^{52,64}$, or photosynthetic pathway (C3 vs. C4) $)^{27,65}$, with contrasting responses to different environmental stressors ${ }^{66,67}$. Clearly, integrating and disentangling the relationship 
between SIF and GPP across species, space, time and in response to environmental stress, remains still a challenge that calls for comprehensive field studies.

\section{Roadmap towards a consistent interpretation of SIF}

The time for multiscale SIF measurements is already here (Fig. 3). Yet, converting these data into meaningful information and new applications still requires effort dedicated to scaling and standardizing methods for SIF interpretation, with particular attention to the seven challenges described above. This process requires accounting for the influence of 1) instrumental, 2) atmospheric, 3) structural and 4) physiological factors to unlock the quantitative association between measured SIF and photosynthesis (Fig. 4). Addressing these challenges requires new data, protocols and models to interpret SIF and bridge the gap between molecular processes, i.e. photosynthesis, and satellite imagery.

At the leaf level, new instruments and techniques employing optical bandpass filters have been developed to record fluorescence spectral dynamics under both natural or controlled illumination, temperature, and $\mathrm{CO}_{2}$ concentration ${ }^{16,68-71}$. Such spectral approaches, combined with foliar pigment analysis, photosynthetic gas exchange, and PAM ChlaF measurements, provide new insights into the connection between SIF and photosynthesis dynamics of leaves ${ }^{42,69,72}$. Going forward, mechanistically modeling the link between SIF and GPP (Challenges 1-4) will require the combination of field campaigns covering full growing seasons, multiple species and stress responses with detailed experimentation under highly controlled conditions, for example using Arabidopsis mutants with altered photochemical properties ${ }^{9,73}$. In particular, the development of versatile field instrumentation and protocols for the estimation of APARg (Challenge 1), energy distribution between PSII and PSI - including the ChlaF contribution from PSI - (Challenge 2), or the quantification of alternative energy sinks (Challenge 4), is key to resolving the spatial and temporal influences of these factors on SIF. 
The synergistic use of complementary data streams can also help to constrain the modelling of photosynthesis and support SIF interpretation. For example, leaf and canopy reflectance data can inform us on the chlorophyll content in the leaf or the amount of leaves in the canopy ${ }^{74}$, relating to $\mathrm{APAR}_{\mathrm{g}}$ (Challenge 1). In addition, reflectance data have been used to explore the regulatory dynamics of $\mathrm{NPQ}^{75}$, which could contribute to resolving energy partitioning in PSII (Challenge 3). This approach is feasible due to the spectral change that accompanies the operation of the xanthophyll cycle ${ }^{76}$ - by which violaxanthin is converted to antheraxanthin and zeaxanthin in a process that modulates $\mathrm{NPQ}^{38,77}$ - as well as the seasonal dynamics of leaf carotenoid and chlorophyll contents ${ }^{78}$. These spectral changes, which have been captured by the photochemical reflectance index $(\mathrm{PRI})^{77,79}$ or the Chlorophyll/Carotenoid Index $(\mathrm{CCI})^{80}$, are now being revisited and investigated in depth across the whole VIS-NIR region alongside with SIF dynamics ${ }^{71,76}$. Clearly, as in the case of SIF, careful use of canopy and atmospheric RTMs will be needed to disentangle these subtle physiologically-induced reflectance changes from those of a dynamic background $^{55}$. In addition to synergies with spectral reflectance, use of thermal imaging ${ }^{81}, \operatorname{radar}^{82}$, or multispectral laser scanning methods ${ }^{83}$ offer interesting possibilities to constrain the carbon reactions of photosynthesis by providing independent information on plant water status (Challenge 4). Likewise, leaf and ecosystem-level measurements of carbonyl sulfide (COS) uptake by vegetation can provide an independent source of information on stomatal conductance in vascular plants $^{84}$, which could be highly relevant for the development and validation of ecosystem-level SIFGPP models.

Process-based and radiative transfer models are required to integrate physical and physiological mechanisms operating at different scales (Challenge 7), providing excellent frameworks for multidisciplinary collaborations to connect molecular-level with Earth-system processes. Clearly, as our mechanistic understanding of the connection between SIF and GPP increases (Challenges 1-4), so will the accuracy of process-based models. For example, the integration of the Farquhar- 
275 Caemmerer-Berry ${ }^{85}$ biochemical model of photosynthesis into dynamic land-surface models (e.g., $276 \mathrm{ORCHIDEE}^{86}$ or BETHY ${ }^{87}$ ) provides a gateway for assimilating satellite SIF data and improving 277 the accuracy of GPP estimations ${ }^{88,89}$. In addition, SIF resides at the core of a new generation of 278 photosynthesis models that emphasize the light reactions ${ }^{45,90}$. In the case of RTMs with established 279 SIF capabilities (Box 2), further improvements can be achieved by coupling with new techniques measuring detailed 3D structures. Leaf RTMs would benefit from including variations in leaf morphology, thylakoid structure, or the spectral signatures of PSI and PSII. The 3D parameterization of canopy RTMs via lidar-based reconstruction methods ${ }^{91,92}$, coupled to nonimaging ${ }^{17,19}$ and imaging proximal/airborne SIF measurements (Fig. 3) ${ }^{93,94}$, offers excellent opportunities to integrate and resolve the diversity of factors that control SIF across space and time (Challenge 7). Drone-based measurements could serve to investigate and model the impact of atmospheric properties on SIF retrieval approaches, by hovering at different distances above the $\operatorname{target}^{54}$ (Challenge 6). Finally, less accurate but simpler alternative methods for separating the physiological and structural influences on the SIF signal have been recently proposed based on the theory of vegetation canopy near-infrared spectral invariants ${ }^{95,96}$. Whether this or other correction methods are applicable to canopy SIF acquisitions across scales, especially observations at very high spatial resolutions (Fig. 3) should be further investigated.

Equally critical for the consistent interpretation of SIF is the establishment of a global network and database of leaf and ecosystem-level SIF measurements covering different biomes, and supporting model development as well as airborne and satellite calibration/validation activities. While regional SIF networks are starting to emerge in North America, Europe, and Asia, their global connectivity should be a priority to promote the adoption of standards for instrument calibrations and long-term monitoring operations (Fig. 4).

Our roadmap for resolving the seven SIF challenges will only succeed through multidisciplinary collaboration involving specialists from across molecular biology, plant physiology, optical physics 
and remote sensing. Together, the characterization and modeling of the interplay between structural, optical and functional dynamics of leaves and plant canopies, can turn our crops and forests into observable field laboratories.

\section{Emerging and potential SIF applications}

Satellite SIF data are already providing new insight into photosynthetic dynamics at the global scale $^{97,98}$. Likewise, with the advent of multiscale SIF measurements (Fig. 3), and as the remaining challenges are overcome (Fig. 4), a new range of SIF applications unfolds across fields of biochemistry, biophysics, ecology, ecophysiology, biogeochemistry, agriculture and forestry (Fig. 5). Equally important, the continuum of scales at which SIF can be measured provides a focal point to promote and strengthen the interaction between research communities, from plant molecular biology to Earth-system science. Here, we outline four examples of potential and emerging SIF applications.

Spatial and $3 D$ photosynthesis. Photosynthetic $\mathrm{CO}_{2}$ assimilation can be measured using infrared gas analyzers, either coupled to chambers or enclosures at the leaf, shoot, and whole-plant level ${ }^{99}$, or with a sonic anemometer at the ecosystem level using the eddy covariance approach ${ }^{100}$. These methods, however, lack detailed spatial information. Spatial measurements of photosynthesis, in terms of photochemical rates of the light reactions, require the use of imaging systems that, to date, have remained restricted to the scale of leaves or small-sized plants, e.g. PAM imaging methods ${ }^{101}$. SIF measurements have potential to fill this scale gap. For example, SIF imaging (Fig. 3) could be benchmarked with eddy-covariance methods to reveal the spatial variability of photosynthesis within the footprint of ecosystem eddy covariance measurements, allowing us to investigate the influence of microenvironment, understory and vertical canopy structure, or the interplay between biological and functional diversity within the ecosystem. Likewise, SIF imaging could be applied to 
resolve photosynthesis dynamics in $3 \mathrm{D}$, helping to advance our understanding of the interaction between plant structure and function ${ }^{102,103}$.

Physiological phenotyping and pre-visual stress detection. Spatial and temporal variations in plant morphological traits (e.g., canopy height, leaf area, and plant growth) have been widely used as markers for field phenotypic variability and to investigate long-term plant stress responses. However, these traits are insufficiently responsive to rapid plant physiological changes. This makes them ill-suited for physiological phenotyping (i.e. breeding plant phenotypes displaying specific physiological responses to the environment), or pre-visual stress detection and subsequent optimization of water, pesticide and fertilizer use. The current phenotyping focus has, therefore, shifted towards measurements in the visible and infrared spectral ranges, where reflectance changes can be associated with specific physiological and biochemical traits ${ }^{104}$ or used for early-stress detection $^{105}$. In this context, emerging SIF imaging systems have already provided promising results for applications in precision agriculture and detection of pest infestations ${ }^{93,106}$. In the near future, these methods could also support precision forestry applications related to seedling production or tree-scale forest management.

Functional plant diversity and spatial ecology. Functional diversity is a fundamental component of the biodiversity concept ${ }^{107}$. As a global network for monitoring biodiversity through remotely sensed plant functional traits is being developed ${ }^{108}$, SIF could become one of the new essential variables for mapping functional diversity across ecosystem and landscape scales, given the wide range of biochemical and physiological factors that SIF is sensitive to (Fig. 2) in relation to plant productivity. For example, SIF has been shown to convey spatial information on leaf mass and chlorophyll content ${ }^{109}$, and other functional plant traits ${ }^{110}$ in various forest ecosystems. Additionally and importantly, the combination of high-resolution structural, spectral and SIF data is potentially the only viable option to investigate ecosystem functions that have remained hidden from our observational abilities, such as photosynthetic phenology in evergreen forests ${ }^{18}$, cryptogamic 
biocrusts $^{111}$ and spatially fragmented Antarctic mosses ${ }^{74}$. Together with spatial photosynthesis, SIF could also offer unique opportunities for studies in spatial ecology ${ }^{111,112}$, where plant environmental responses and biotic interactions could leave their imprint on SIF.

Carbon and water cycle studies. The carbon and water cycles of terrestrial ecosystems are intricately connected via stomatal regulation and total leaf area. Because both canopy evapotranspiration and canopy SIF dynamics are strongly controlled by leaf area, and since ChlaF can also decrease with stomatal closure - via increased NPQ in response to water stress ${ }^{113,114}$; tower and satellite SIF have been preliminarily used to investigate canopy conductance and plant transpiration ${ }^{115,116}$. No doubt, better constraints on transpiration and photosynthetic dynamics in land-surface models will be achieved as the mechanistic basis of SIF is elucidated across scales (Challenges 1-7), and the integration of SIF with other remote sensing datasets increases, such as land-surface temperature ${ }^{115}$, surface soil moisture ${ }^{89}$, radar-measured vegetation optical depth characterizing canopy structure and water content ${ }^{117}$, or column-averaged atmospheric $\mathrm{CO}_{2}{ }^{92}$. New knowledge of photosynthesis at the ecosystem and regional scales will bring further insight into the large-scale interactions between environmental drivers and plant productivity, and feedbacks between the biosphere and atmosphere.

\section{Concluding remarks}

The SIF signal gathers a wealth of physiological, biochemical, and structural information as it travels from the photosystems to the top of canopy and beyond (Fig. 2). This can leave the impression that SIF is, to use the classic analogy, the 'Swiss Army Knife' of photosynthesis measurements. Critically, the variation in SIF caused by physical and biotic factors is entangled in the spatiotemporal domain, and our capacity to disentangle it into useful informative components requires further attention. Historically, photosynthesis research has been a multidisciplinary endeavor, with breakthroughs in the $20^{\text {th }}$ century emerging from collaboration between chemists, 
biologists and physicists. We are now entering a new era of multiscale observations of photosynthesis which requires the interdisciplinary research environment to flourish further, this time to resolve the mechanistic connection between SIF and GPP and to scale it across space and time. The technology to measure SIF is developing at a faster pace than our capacity to interpret the acquired data. With the challenges, roadmap and unfolding opportunities introduced here we hope to encourage more scientists to join the multidisciplinary quest to reveal the true potential of SIF observation.

\section{References}

1. Genty, B., Wonders, J. \& Baker, N. R. Non-photochemical quenching of Fo in leaves is emission wavelength dependent: consequences for quenching analysis and its interpretation. Photosynth. Res. 26, 133-139 (1990).

2. Franck, F., Juneau, P. \& Popovic, R. Resolution of the photosystem I and photosystem II contributions to chlorophyll fluorescence of intact leaves at room temperature. Biochim. Biophys. Acta-Bioenergetics 1556, 239-246 (2002).

3. Neubauer, C. \& Schreiber, U. The polyphasic rise of chlorophyll fluorescence upon onset of strong continuous illumination: I. Saturation characteristics and partial control by the photosystem II acceptor side. Zeitschrift für Naturforschung C 42, 1246-1254 (1987).

4. Strasser, R. J., Tsimilli-Michael, M. \& Srivastava, A. Analysis of the chlorophyll a fluorescence transient. In: Papageorgiou G.C., Govindjee (eds) Chlorophyll a Fluorescence. Advances in Photosynthesis and Respiration, vol 19. Springer, Dordrecht (2004).

5. Schreiber, U., Schliwa, U. \& Bilger, W. Continuous recording of photochemical and nonphotochemical chlorophyll fluorescence quenching with a new type of modulation fluorometer. Photosynth. Res. 10, 51-62 (1986). 
6. Maxwell, K. \& Johnson, G. N. Chlorophyll fluorescence-a practical guide. J. Exp. Bot. 51, 659-668 (2000).

7. Govindjee, E. 63 Years since Kautsky-chlorophyll-a fluorescence. Aust. J. Plant Physiol. 22,

9. Tikkanen, M., Rantala, S., Grieco, M. \& Aro, E. Comparative analysis of mutant plants

8. Porcar-Castell, A. et al. Linking chlorophyll a fluorescence to photosynthesis for remote sensing applications: mechanisms and challenges. J. Exp. Bot. 65, 4065-4095 (2014). impaired in the main regulatory mechanisms of photosynthetic light reactions-From biophysical measurements to molecular mechanisms. Plant Physiol. Biochem. 112, 290-301 (2017).

10. Kolber, Z. et al. Measuring photosynthetic parameters at a distance: laser induced fluorescence transient (LIFT) method for remote measurements of photosynthesis in terrestrial vegetation. Photosynth. Res. 84, 121-129 (2005).

11. Keller, B. et al. Genotype specific photosynthesis x environment interactions captured by automated fluorescence canopy scans over two fluctuating growing seasons. Front. Plant Sci. 10, 1482 (2019).

12. Mohammed, G. H. et al. Remote sensing of solar-induced chlorophyll fluorescence (SIF) in vegetation: 50 years of progress. Remote Sens. Environ. 231, 111177 (2019).

13. Evain, S., Camenen, L. \& Moya, I. Three-channel detector for remote sensing of chlorophyll fluorescence and reflectance from vegetation. In: M. Leroy (ed.), $8^{\text {th }}$ International symposium: physical measurements and signatures in remote sensing, pp. 395-400. Aussois, CNES, France (2001).

14. Louis, J. et al. Remote sensing of sunlight-induced chlorophyll fluorescence and reflectance of Scots pine in the boreal forest during spring recovery. Remote Sens. Environ. 96, 37-48 (2005). 
15. Guanter, L. et al. Estimation of solar-induced vegetation fluorescence from space measurements. Geophys. Res. Lett. 34 (2007).

16. Aasen, H. et al. Sun-induced chlorophyll fluorescence II: review of passive measurement setups, protocols, and their application at the leaf to canopy level. Remote Sensing 11, 927 (2019)

17. Yang, X. et al. Solar-induced chlorophyll fluorescence that correlates with canopy photosynthesis on diurnal and seasonal scales in a temperate deciduous forest. Geophys. Res. Lett. 42, 2977-2987 (2015).

18. Magney, T. S. et al. Mechanistic evidence for tracking the seasonality of photosynthesis with solar-induced fluorescence. PNAS 116, 11640-11645 (2019).

19. Bendig, J., Malenovský, Z., Gautam, D. \& Lucieer, A. Solar-Induced Chlorophyll Fluorescence Measured From an Unmanned Aircraft System: Sensor Etaloning and Platform Motion Correction. IEEE Trans. Geosci. Remote Sens. (2019).

20. Vargas, J. Q. et al. Unmanned aerial systems (UAS)-based methods for solar induced chlorophyll fluorescence (SIF) retrieval with non-imaging spectrometers: state of the art. Remote Sens. 12, 1624 (2020).

21. Rascher, U. et al. Sun-induced fluorescence-a new probe of photosynthesis: First maps from the imaging spectrometer HyPlant. Global Change Biol. 21, 4673-4684 (2015).

22. Frankenberg, C. et al. The Chlorophyll Fluorescence Imaging Spectrometer (CFIS), mapping far red fluorescence from aircraft. Remote Sens. Environ. 217, 523-536 (2018).

23. Frankenberg, C. et al. New global observations of the terrestrial carbon cycle from GOSAT: Patterns of plant fluorescence with gross primary productivity. Geophys. Res. Lett. 38 (2011).

24. Köhler, P. et al. Global Retrievals of Solar-Induced Chlorophyll Fluorescence at Red Wavelengths With TROPOMI. Geophys. Res. Lett. 47, e2020GL087541 (2020). 
25. Drusch, M. et al. The fluorescence explorer mission concept - ESA's earth explorer 8. IEEE Trans. Geosci. Remote Sens. 55, 1273-1284 (2016).

26. Olascoaga, B., Mac Arthur, A., Atherton, J. \& Porcar-Castell, A. A comparison of methods to estimate photosynthetic light absorption in leaves with contrasting morphology. Tree Physiol. 36, 368-379 (2016).

27. Zhang, Z. et al. Assessing bi-directional effects on the diurnal cycle of measured solarinduced chlorophyll fluorescence in crop canopies. Agric. For. Meteorol. 295, 108147 (2020).

28. Bittner, T., Irrgang, K., Renger, G. \& Wasielewski, M. R. Ultrafast excitation energy transfer and exciton-exciton annihilation processes in isolated light harvesting complexes of photosystem II (LHC II) from spinach. J. Phys. Chem. 98, 11821-11826 (1994).

29. Kalaji, H. M. et al. Frequently asked questions about chlorophyll fluorescence, the sequel. Photosynth. Res. 132, 13-66 (2017).

30. Genty, B., Briantais, J. \& Baker, N. R. The relationship between the quantum yield of photosynthetic electron transport and quenching of chlorophyll fluorescence. Biochim. Biophys. Acta-General Subjects 990, 87-92 (1989).

31. Anderson, J. M., Chow, W. S. \& Goodchild, D. J. Thylakoid membrane organisation in sun/shade acclimation. Funct. Plant Biol. 15, 11-26 (1988).

32. Ballottari, M., Dall'Osto, L., Morosinotto, T. \& Bassi, R. Contrasting behavior of higher plant photosystem I and II antenna systems during acclimation. J. Biol. Chem. 282, 89478958 (2007).

33. Schreiber, U., Klughammer, C. \& Kolbowski, J. Assessment of wavelength-dependent parameters of photosynthetic electron transport with a new type of multi-color PAM chlorophyll fluorometer. Photosynth. Res. 113, 127-144 (2012). 
34. Laisk, A. et al. A computer-operated routine of gas exchange and optical measurements to diagnose photosynthetic apparatus in leaves. Plant, Cell Environ. 25, 923-943 (2002).

35. Pfündel, E. Estimating the contribution of photosystem I to total leaf chlorophyll fluorescence. Photosynthesis Res. 56, 185-195 (1998).

36. Peterson, R. B. et al. Fluorescence Fo of photosystems II and I in developing C3 and C4 leaves, and implications on regulation of excitation balance. Photosynth. Res. 122, 41-56

37. Pfündel, E. E. Simultaneously measuring pulse-amplitude-modulated (PAM) chlorophyll fluorescence of leaves at wavelengths shorter and longer than $700 \mathrm{~nm}$. Photosynth. Res., 1$14(2021)$.

38. Demmig-Adams, B. \& Adams III, W. W. Photoprotection in an ecological context: the remarkable complexity of thermal energy dissipation. New Phytol. 172, 11-21 (2006).

39. Porcar-Castell, A. A high-resolution portrait of the annual dynamics of photochemical and non-photochemical quenching in needles of Pinus sylvestris. Physiol. Plant. 143, 139-153 (2011).

40. Van der Tol, C., Berry, J. A., Campbell, P. \& Rascher, U. Models of fluorescence and photosynthesis for interpreting measurements of solar-induced chlorophyll fluorescence. $J$. Geophys. Res.: Biogeosciences 119, 2312-2327 (2014).

41. Springer, K. R., Wang, R. \& Gamon, J. A. Parallel seasonal patterns of photosynthesis, fluorescence, and reflectance indices in boreal trees. Remote Sens. 9, 691 (2017).

42. Zhang, C. et al. Do all chlorophyll fluorescence emission wavelengths capture the spring recovery of photosynthesis in boreal evergreen foliage? Plant, Cell Environ. 42, 3264-3279 (2019).

43. Ensminger, I. et al. Intermittent low temperatures constrain spring recovery of photosynthesis in boreal Scots pine forests. Glob. Change Biol. 10, 995-1008 (2004). 
44. Verhoeven, A. Sustained energy dissipation in winter evergreens. New Phytol. 201, 57-65 (2014).

45. Gu, L., Han, J., Wood, J. D., Chang, C. Y. \& Sun, Y. Sun-induced Chl fluorescence and its importance for biophysical modeling of photosynthesis based on light reactions. New Phytol. 223, 1179-1191 (2019).

46. Raczka, B. et al. Sustained nonphotochemical quenching shapes the seasonal pattern of solar-induced fluorescence at a high-elevation evergreen forest. J. Geophys. Res.: Biogeosciences 124, 2005-2020 (2019).

47. Nixon, P. J. Chlororespiration. Philos. Trans. R. Soc. Lond., B, Biol. Sci. 355, 1541-1547 (2000).

48. Ogren, W. L. Photorespiration: pathways, regulation, and modification. Annu. Rev. Plant Physiol. 35, 415-442 (1984).

49. Asada, K. The water-water cycle in chloroplasts: scavenging of active oxygens and dissipation of excess photons. Annu. Rev. Plant Biol. 50, 601-639 (1999).

50. Morfopoulos, C. et al. A model of plant isoprene emission based on available reducing power captures responses to atmospheric CO2. New Phytol. 203, 125-139 (2014).

51. Maseyk, K., Lin, T., Cochavi, A., Schwartz, A. \& Yakir, D. Quantification of leaf-scale light energy allocation and photoprotection processes in a Mediterranean pine forest under extensive seasonal drought. Tree Physiol. 39, 1767-1782 (2019).

52. Migliavacca, M. et al. Plant functional traits and canopy structure control the relationship between photosynthetic $\mathrm{CO}_{2}$ uptake and far-red sun-induced fluorescence in a Mediterranean grassland under different nutrient availability. New Phytol. 214, 1078-1091 (2017).

53. Kallel, A. FluLCVRT: Reflectance and fluorescence of leaf and canopy modeling based on Monte Carlo vector radiative transfer simulation. J. Quant. Spectrosc. Radiat. Transf. 253, $107183(2020)$ 
54. Sabater, N. et al. Compensation of oxygen transmittance effects for proximal sensing retrieval of canopy-leaving sun-induced chlorophyll fluorescence. Remote Sens. 10, 1551 (2018).

55. Sabater, N., Kolmonen, P., Van Wittenberghe, S., Arola, A. \& Moreno, J. Challenges in the atmospheric characterization for the retrieval of spectrally resolved fluorescence and PRI region dynamics from space. Remote Sens. Environ. 254, 112226 (2021).

56. Iermak, I., Vink, J., Bader, A. N., Wientjes, E. \& van Amerongen, H. Visualizing heterogeneity of photosynthetic properties of plant leaves with two-photon fluorescence lifetime imaging microscopy. Biochim. Biophys. Acta-Bioenergetics 1857, 1473-1478 (2016).

57. Romero, J. M., Cordon, G. B. \& Lagorio, M. G. Modeling re-absorption of fluorescence from the leaf to the canopy level. Remote Sens. Environ. 204, 138-146 (2018).

58. Magney, T. S. et al. Disentangling changes in the spectral shape of chlorophyll fluorescence: Implications for remote sensing of photosynthesis. J. Geophys. Res.: Biogeosciences 124, 1491-1507 (2019).

59. Murchie, E. H. et al. Measuring the dynamic photosynthome. Ann. Bot. 122, 207-220 (2018).

60. Magney, T. S., Barnes, M. L. \& Yang, X. On the covariation of chlorophyll fluorescence and photosynthesis across scales. Geophys. Res. Lett. 47, e2020GL091098 (2020).

61. Yang, P., van der Tol, C., Campbell, P. K. \& Middleton, E. M. Unraveling the physical and physiological basis for the solar-induced chlorophyll fluorescence and photosynthesis relationship using continuous leaf and canopy measurements of a corn crop. Biogeosciences 18, 441-465 (2021).

62. Liu, X. et al. Downscaling of solar-induced chlorophyll fluorescence from canopy level to photosystem level using a random forest model. Remote Sens. Environ. 231, 110772 (2019). 
63. Joiner, J. et al. Systematic Orbital Geometry-Dependent Variations in Satellite SolarInduced Fluorescence (SIF) Retrievals. Remote Sens.12, 2346 (2020).

64. Dechant, B. et al. Canopy structure explains the relationship between photosynthesis and sun-induced chlorophyll fluorescence in crops. Remote Sens. Environ. 241, 111733 (2020).

65. He, L. et al. From the Ground to Space: Using Solar-Induced Chlorophyll Fluorescence to Estimate Crop Productivity. Geophys. Res. Lett. 47, e2020GL087474 (2020).

66. Ač, A. et al. Meta-analysis assessing potential of steady-state chlorophyll fluorescence for remote sensing detection of plant water, temperature and nitrogen stress. Remote Sens. Environ. 168, 420-436 (2015).

67. Wohlfahrt, G. et al. Sun-induced fluorescence and gross primary productivity during a heat wave. Sci. Rep. 8, 1-9 (2018).

68. Van Wittenberghe, S., Alonso, L., Verrelst, J., Moreno, J. \& Samson, R. Bidirectional suninduced chlorophyll fluorescence emission is influenced by leaf structure and light scattering properties: A bottom-up approach. Remote Sens. Environ. 158, 169-179 (2015).

69. Magney, T. S. et al. Connecting active to passive fluorescence with photosynthesis: A method for evaluating remote sensing measurements of Chl fluorescence. New Phytol. 215, 1594-1608 (2017).

70. Rajewicz, P. A., Atherton, J., Alonso, L. \& Porcar-Castell, A. Leaf-level spectral fluorescence measurements: comparing methodologies for broadleaves and needles. Remote Sens. 11, 532 (2019).

71. Van Wittenberghe, S., Alonso, L., Malenovský, Z. \& Moreno, J. In vivo photoprotection mechanisms observed from leaf spectral absorbance changes showing VIS-NIR slowinduced conformational pigment bed changes. Photosynth. Res. 142, 283-305 (2019). 
72. Meeker, E. W., Magney, T. S., Bambach, N., Momayyezi, M. \& McElrone, A. J. Modification of a gas exchange system to measure active and passive chlorophyll fluorescence simultaneously under field conditions. AoB Plants 13, plaa066 (2021).

73. Acebron, K. et al. Diurnal dynamics of nonphotochemical quenching in Arabidopsis npq mutants assessed by solar-induced fluorescence and reflectance measurements in the field. New Phytol. (2020).

74. Malenovský, Z., Lucieer, A., King, D. H., Turnbull, J. D. \& Robinson, S. A. Unmanned aircraft system advances health mapping of fragile polar vegetation. Methods Ecol. Evol. 8, 1842-1857 (2017).

75. Atherton, J., Nichol, C. J. \& Porcar-Castell, A. Using spectral chlorophyll fluorescence and the photochemical reflectance index to predict physiological dynamics. Remote Sens. Environ. 176, 17-30 (2016).

76. Van Wittenberghe, S. et al. Combined dynamics of the 500-600 nm leaf absorption and chlorophyll fluorescence changes in vivo: Evidence for the multifunctional energy quenching role of xanthophylls. Biochim. Biophys. Acta-Bioenergetics 1862, 148351 (2021).

77. Gamon, J. A. et al. Remote sensing of the xanthophyll cycle and chlorophyll fluorescence in sunflower leaves and canopies. Oecologia 85, 1-7 (1990).

78. Filella, I. et al. PRI assessment of long-term changes in carotenoids/chlorophyll ratio and short-term changes in de-epoxidation state of the xanthophyll cycle. Int. J. Remote Sens. 30, 4443-4455 (2009).

79. Peñuelas, J., Filella, I. \& Gamon, J. A. Assessment of photosynthetic radiation-use efficiency with spectral reflectance. New Phytol. 131, 291-296 (1995).

80. Gamon, J. A. et al. A remotely sensed pigment index reveals photosynthetic phenology in evergreen conifers. PNAS 113, 13087-13092 (2016). 
81. Costa, J. M., Grant, O. M. \& Chaves, M. M. Thermography to explore plant-environment interactions. J. Exp. Bot. 64, 3937-3949 (2013).

82. Konings, A. G., Rao, K. \& Steele-Dunne, S. C. Macro to micro: microwave remote sensing of plant water content for physiology and ecology. New Phytol. 223, 1166-1172 (2019).

83. Junttila, S. et al. Terrestrial laser scanning intensity captures diurnal variation in leaf water potential. Remote Sens. Environ. 255, 112274 (2021).

84. Whelan, M. E. et al. Two Scientific Communities Striving for a Common Cause: innovations in carbon cycle science. Bull. Am. Meteorol. Soc. (2020).

85. Farquhar, G. D., von Caemmerer, S. v. \& Berry, J. A. A biochemical model of photosynthetic CO 2 assimilation in leaves of C 3 species. Planta 149, 78-90 (1980).

86. Bacour, C. et al. Improving estimates of gross primary productivity by assimilating solarinduced fluorescence satellite retrievals in a terrestrial biosphere model using a processbased SIF model. J. Geophys. Res.: Biogeosciences 124, 3281-3306 (2019).

87. Norton, A. J. et al. Estimating global gross primary productivity using chlorophyll fluorescence and a data assimilation system with the BETHY-SCOPE model. Biogeosciences 16, 3069-3093 (2019).

88. Thum, T. et al. Modelling sun-induced fluorescence and photosynthesis with a land surface model at local and regional scales in northern Europe. Biogeosciences 14, 1969-1987 (2017).

89. Qiu, B., Chen, J. M., Ju, W., Zhang, Q. \& Zhang, Y. Simulating emission and scattering of solar-induced chlorophyll fluorescence at far-red band in global vegetation with different canopy structures. Remote Sens. Environ. 233, 111373 (2019).

90. Johnson, J. E. \& Berry, J. A. The role of Cytochrome b $6 \mathrm{f}$ in the control of steady-state photosynthesis: a conceptual and quantitative model. Photosynth. Res., 1-36 (2021). 
91. Janoutová, R. et al. Influence of 3D spruce tree representation on accuracy of airborne and satellite forest reflectance simulated in DART. Forests 10, 292 (2019).

92. Liu, W. et al. Simulating solar-induced chlorophyll fluorescence in a boreal forest stand reconstructed from terrestrial laser scanning measurements. Remote Sens. Environ. 232, 111274 (2019).

93. Pinto, F. et al. Sun-induced chlorophyll fluorescence from high-resolution imaging spectroscopy data to quantify spatio-temporal patterns of photosynthetic function in crop canopies. Plant, Cell Environ. 39, 1500-1512 (2016).

94. Siegmann, B. et al. The high-performance airborne imaging spectrometer HyPlant-From raw images to top-of-canopy reflectance and fluorescence products: Introduction of an automatized processing chain. Remote Sens. 11, 2760 (2019).

95. Yang, P., van der Tol, C., Campbell, P. K. \& Middleton, E. M. Fluorescence Correction Vegetation Index (FCVI): A physically based reflectance index to separate physiological and non-physiological information in far-red sun-induced chlorophyll fluorescence. Remote Sens. Environ. 240, 111676 (2020).

96. Zeng, Y. et al. A radiative transfer model for solar induced fluorescence using spectral invariants theory. Remote Sens. Environ. 240, 111678 (2020).

97. Green, J. K. et al. Large influence of soil moisture on long-term terrestrial carbon uptake. Nature 565, 476 (2019).

98. Wang, S. et al. Urban - rural gradients reveal joint control of elevated CO 2 and temperature on extended photosynthetic seasons. Nat. Ecol. Evo. 3, 1076-1085 (2019).

99. Long, S. P., Farage, P. K. \& Garcia, R. L. Measurement of leaf and canopy photosynthetic CO2 exchange in the field. J. Exp. Bot. 47, 1629-1642 (1996). 
100. Baldocchi, D. D. Assessing the eddy covariance technique for evaluating carbon dioxide exchange rates of ecosystems: past, present and future. Glob. Change Biol. 9, 479-492 (2003).

101. Kaiser, Y. I., Menegat, A. \& Gerhards, R. Chlorophyll fluorescence imaging: a new method for rapid detection of herbicide resistance in A lopecurus myosuroides. Weed Res. 53, 399$406(2013)$.

102. Sievänen, R., Godin, C., DeJong, T. M. \& Nikinmaa, E. Functional-structural plant models: a growing paradigm for plant studies. Ann. Bot. 114, 599-603 (2014).

103. Damm, A., Paul-Limoges, E., Kükenbrink, D., Bachofen, C. \& Morsdorf, F. Remote sensing of forest gas exchange: Considerations derived from a tomographic perspective. Glob. Change Biol. 26, 2717-2727 (2020).

104. Ensminger, I. Fast track diagnostics: Hyperspectral reflectance differentiates disease from drought stress in trees. Tree Physiol. 40, 1143-1146 (2020).

105. Mutka, A. M. \& Bart, R. S. Image-based phenotyping of plant disease symptoms. Frontiers in plant science 5, 734 (2015).

106. Zarco-Tejada, P. J. et al. Previsual symptoms of Xylella fastidiosa infection revealed in spectral plant-trait alterations. Nat. Plants 4, 432-439 (2018).

107. Díaz, S. \& Cabido, M. Vive la différence: plant functional diversity matters to ecosystem processes. Trends Ecol. Evol. 16, 646-655 (2001).

108. Skidmore, A. K. et al. Environmental science: Agree on biodiversity metrics to track from space. Nat. News 523, 403 (2015).

109. Tagliabue, G. et al. Sun-induced fluorescence heterogeneity as a measure of functional diversity. Remote Sens. Environ. 247, 111934 (2020). 
110. Pacheco-Labrador, J. et al. Multiple-constraint inversion of SCOPE. Evaluating the potential of GPP and SIF for the retrieval of plant functional traits. Remote Sens. Environ. 234, 111362 (2019).

111. Smith, W. K. et al. Remote sensing of dryland ecosystem structure and function: Progress, challenges, and opportunities. Remote Sens. Environ. 233, 111401 (2019).

112. Kellner, J. R., Albert, L. P., Burley, J. T. \& Cushman, K. C. The case for remote sensing of individual plants. Am. J. Bot. 106, 1139-1142 (2019).

113. Flexas, J. et al. Steady-state chlorophyll fluorescence (Fs) measurements as a tool to follow variations of net $\mathrm{CO} 2$ assimilation and stomatal conductance during water-stress in $\mathrm{C} 3$ plants. Physiol. Plant. 114, 231-240 (2002).

114. Marrs, J. K. et al. Solar-induced fluorescence does not track photosynthetic carbon assimilation following induced stomatal closure. Geophys. Res. Lett. 47, e2020GL087956 (2020).

115. Maes, W. H. et al. Sun-induced fluorescence closely linked to ecosystem transpiration as evidenced by satellite data and radiative transfer models. Remote Sens. Environ. 249, $112030(2020)$.

116. Shan, N. et al. A model for estimating transpiration from remotely sensed solar-induced chlorophyll fluorescence. Remote Sens. Environ. 252, 112134 (2021).

117. Liu, W. et al. Simulating solar-induced chlorophyll fluorescence in a boreal forest stand reconstructed from terrestrial laser scanning measurements. Remote Sens. Environ. 232, 111274 (2019).

118. Albert, L. P. et al. Stray light characterization in a high-resolution imaging spectrometer designed for solar-induced fluorescence. Proc. SPIE 10986, Algorithms, Technologies, and Applications for Multispectral and Hyperspectral Imagery XXV, 109860G (2019). 
119. Meroni, M. et al. Remote sensing of solar-induced chlorophyll fluorescence: Review of methods and applications. Remote Sens. Environ. 113, 2037-2051 (2009).

120. Cendrero-Mateo, M. P. et al. Sun-induced chlorophyll fluorescence III: Benchmarking retrieval methods and sensor characteristics for proximal sensing. Remote Sens. 11, 962 (2019).

121. Vilfan, N. et al. Extending Fluspect to simulate xanthophyll driven leaf reflectance dynamics. Remote Sens. Environ. 211, 345-356 (2018).

122. Yang, P., Prikaziuk, E., Verhoef, W. \& van der Tol, C. SCOPE 2.0: A model to simulate vegetated land surface fluxes and satellite signals. Geosci. Model Dev. Discuss., 1-26 (2020).

123. Gastellu-Etchegorry, J. et al. DART: recent advances in remote sensing data modeling with atmosphere, polarization, and chlorophyll fluorescence. IEEE J. Sel. Top. Appl. Earth Obs. Remote Sens. 10, 2640-2649 (2017).

Corresponding author: Albert Porcar-Castell (joan.porcar@helsinki.fi)

Acknowledgements: This perspective idea originated during the Fluorescence Across Space and Time (FAST) Workshop, which took place in Hyytiälä Forestry Research Station (SMEARII, Finland) during February 2019. We thank the following participants for active discussions during the workshop: Juliane Bendig, Kukka-Maaria Erkkilä, Noda Hibiki, Laura V. Junker-Frohn, Valentyna Kuznetsova, Hannakaisa Lindqvist, Paul Näthe, Jaakko Oivukkamaki, Neus Sabater, Twinkle Solanki, Tea Thum, Shan Xu and Chao Zhang. We also thank Barry Osmond and Josep Peñuelas for valuable comments to the manuscript, to Nuria Altimir for improving graphic design of Figs 1 and 5, and to Bastian Siegmann for the preparation of the HyPlant image in Fig. 3. The 
709

710

711

Academy of Finland (Project \# 288039 and 319211) is acknowledged for the financial support. ZM was supported by the Australian Research Council (FT160100477), TM was supported by the National Aeronautics and Space Administration (80NSSC19M0129), and SVW was supported by the Generalitat Valenciana and the European Social Fund (APOSTD/2018/162). Headwall SIF images from LPA and JRK were supported by grants from the Institute at Brown for Environment and Society at Brown University.

Author contributions: APC conceived the original idea and wrote the manuscript with ZM, TM, BL, SVW, BFM, FM, YZ, KM with comments and contributions from all co-authors. In addition, these authors had special contribution to the following parts: Fig.1 (APC, ZM and SVW), Fig.2 (APC, BFM, TM and SVW), Fig. 3 (LPA, UR and JRK), Fig. 4. (APC, ZM, UR, BFM), Fig. 5 (JIGP, JA, ZM, IE), Box 1 (TM, APC), Box 2 (ZM, APC), Supplementary information (ZM, FZ). 


\section{Figure Legends}

Figure 1. From incoming radiation to observed SIF and photosynthesis: mechanistic challenges. Solar radiation reaching the top of the atmosphere (TOA) is partly absorbed and scattered by atmospheric gases and particles, decreasing its intensity as it reaches the bottom of the atmosphere (BOA), generating specific absorption features. Part of the radiation is absorbed by photosynthetic pigments in vegetation and leaves (fAPARg) (Challenge 1), associated with either photosystem I (PSI) or photosystem II (PSII), which contribute with differential dynamics and spectral properties to overall SIF emission (Challenge 2). Within each photosystem, energy is further partitioned into three dynamic processes (Challenge 3): i) photochemistry (leading mainly to linear (LET) or cyclic (CET) electron transport, the latter involving PSI only), ii) thermal energy dissipation, and iii) ChlaF. Photosynthetic energy (expressed for simplicity in terms of NADPH equivalents) is further partitioned between alternative energy sinks and gross photosynthesis (AG), and again between gross primary productivity (GPP) and photorespiration $\left(\mathrm{P}_{\mathrm{R}}\right)$, with dynamics that are not necessarily seen by SIF (Challenge 4). Notably, because it is only possible to measure the net flux of $\mathrm{CO}_{2}$ from a leaf or ecosystem, i.e. net photosynthesis or net primary productivity (NPP), the rate of daytime respiration $\left(\mathrm{R}_{\mathrm{D}}\right)$ must be known or estimated. In turn, because emitted ChlaF overlaps with the absorption spectra of leaves and plant canopies, some SIF photons - especially those in the red wavelengths - are re-absorbed within the canopy (Challenge 5). Emitted ChlaF is further scattered and absorbed by aerosols and gases in the atmosphere (Challenge 6).

\section{Figure 2. The connection between SIF and GPP across space and time. The relationship} between SIF and GPP is affected by multiple factors as we move across spatial and temporal scales. Some factors exert a similar effect on SIF and GPP, keeping them positively correlated - we call these couplers. Other factors differentially affect SIF and GPP - we call these decouplers. Factors 
driving the dynamics of NPQ and APAR will tend to keep SIF and GPP coupled both across space and time, whereas factors adding variation to the energy partitioning between ChlF and GPP, or influencing the reabsorption of ChlF, will tend to decouple SIF from GPP (see examples in the figure). Note how the shape of the ChlF spectrum ("Spectral dynamics") changes across scales in response to reabsorption within the chloroplast, leaf and canopy, measurable as SIF only within discrete wavelengths at the canopy and ecosystem levels (Box 1). Equally important to our understanding of the spatial context of the factors that couple/decouple SIF to GPP is understanding their temporal range of action (lower panels). For example, the rapid (second/minute) decrease in ChlaF upon saturating illumination of dark acclimated leaves reflects the dynamics of $\mathrm{NPQ}^{76}$. Similar dynamics can be seen under natural conditions at the diurnal/seasonal scale in Scots pine needles, as the quantum yield of fluorescence $(\Phi F)$ responds to PQ and NPQ (redrawn from PorcarCastell $^{39}$ ). Here, SIF was estimated for illustrative purposes as SIF (r.u.) = PAR x 0.8 x 0.5 x $\Phi F$, where 0.8 and 0.5 are estimates for fAPARg and the fraction of radiation absorbed by PSII. Likewise, interannual dynamics at the regional-to-local scales ${ }^{24}$ can reflect changes in canopy structure, physiological stress responses or other functional traits. Ultimately, the challenge of integrating and disentangling the impact of these couplers/decouplers across space, time, species and plant stress responses remains (Challenge 7).

Figure 3. State-of-the-art SIF imaging methods allow for the observation of SIF across a continuum of scales: from the leaf-to-individual (top row) to the individual-to-landscape (bottom row). Panel A shows an RGB image of a senescing maple tree next to an oak tree with green leaves. Panel B shows the SIF image of the same trees retrieved in the O2A band at $760 \mathrm{~nm}$ (SIF760) using a commercial, off-the-shelf imaging spectrometer ${ }^{118}$ mounted on a tripod some meters away and after applying a filter to exclude non-vegetation pixels (pixels with an normalized difference vegetation index $($ NDVI) $<0.65)$. As expected, the green and photosynthetically active oak emitted SIF at 
higher magnitude (Panel C) than the senescing maple. Similarly, panels D-E present an airborne RGB and SIF760 map obtained with data from the HyPlant sensor collected at an altitude of $680 \mathrm{~m}$ above ground ${ }^{94}$. The scene shows several plots within an experimental apple tree plantation at the agricultural research site Campus Klein-Altendorf (University of Bonn, Germany), where apple tree varieties of different ages were growing in a typical row structure. Single tree crowns were segmented by overlaying the SIF images with a 3D surface map and all pixels that were related to a background signal (defined as ground level $+30 \mathrm{~cm}$ ) were excluded. The image visualizes the signal of individual trees, where each pixel corresponds to an area of $1 \times 1$ meters and thus the small clusters represent the signal of an individual tree.

Figure 4. A roadmap towards the standardized interpretation of SIF. The critical steps, data sources and methods that will be required to overcome the seven challenges are introduced to allow for a consistent interpretation of spectral observations in terms of leaf, canopy and ecosystem traits.

Figure 5. Potential and emerging SIF applications illustrated in the form of a "SIF-city" metro plan, where different colors denote five fields of plant science. Identified research applications (metro stops) are causally connected in individual communication lines, but the final trajectories and number of stops will depend on how the field of SIF research evolves over the next years. The redcolored stops denote the application topics elaborated in Section 3. 


\section{Box 1 | Principle of solar-induced fluorescence (SIF) retrieval}

SIF measurements take place outdoors, under ambient sunlight. Accordingly, when pointing a spectroradiometer towards a leaf or plant canopy to make a SIF measurement, we face the challenge that vegetation is highly reflective in the near infrared (NIR) wavelengths, and the signal is dominated by reflected light. The retrieval of SIF from the background reflected radiation is made possible thanks to the spectral properties of incoming light.

The solar spectrum, as measured above a plant canopy, is not continuous; rather, radiation is strongly attenuated within so-called Fraunhofer absorption lines and telluric absorption bands originating from absorption by gases in the Sun's photosphere or the Earth's atmosphere, respectively (see Fig. 1 and an idealized spectral feature in A). These features are exploited by the Fraunhofer line depth (FLD) methods ${ }^{119}$ where at least four spectral measurements, usually more ${ }^{120}$, are required: the irradiance of the incoming sunlight and the apparent reflected radiance (called apparent, as it includes also SIF), inside and outside of the spectral absorption feature $\left(\mathrm{E}_{\mathrm{in}} / \mathrm{E}_{\text {out }}\right.$ and $\mathrm{L}_{\mathrm{in}} / \mathrm{L}_{\text {out }}$, respectively). Since SIF contributes photons similarly both inside and outside the spectral feature (B), the relative contribution of SIF to reflected radiation is significantly greater inside the spectral feature, causing an increase in the apparent reflectance $(\mathbf{C})$. This increase is proportional to the amount of SIF and can be used to construct a system of equations to retrieve SIF.

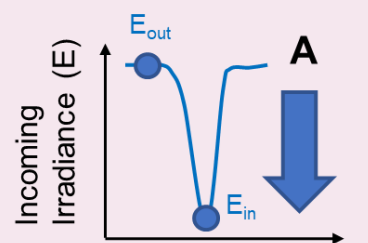

Although not mutually exclusive, SIF measurements are often conducted using either the Fraunhofer or Telluric absorption bands, which involve some tradeoffs:

- Fraunhofer lines (multiple lines across the SIF spectrum). The advantage of these retrievals lies in their lower sensitivity to atmospheric properties, which is practical for remote measurements as well as applications with variable

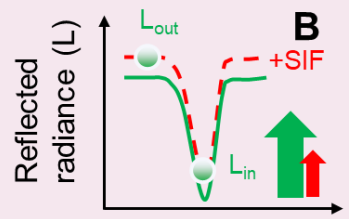
target-to-sensor distances (e.g., multiangular tower measurements). The main disadvantage is that they require spectrometers with extremely high spectral resolutions and generally require longer periods of signal integration.

- Telluric bands (mainly oxygen absorption bands B and A, centered around 687-692 $\mathrm{nm}$; and 759-770 nm, respectively). Since these bands are broader,

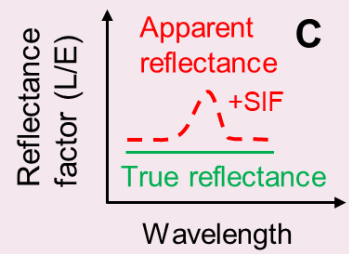
measurements do not require as high spectral resolution and can be also conducted with shorter integration time, which can be especially suitable for some applications (e.g. drone-based observations). Their main disadvantage is that attention must be paid to corrections for atmospheric absorption (Challenge 6). 


\section{Box 2 | Radiative transfer models (RTMs)}

- Forward mode. When the required inputs are provided, RTMs are capable of simulating leaf and canopy SIF together with reflected and emitted optical and thermal radiance. Once successfully validated by independent measurements, RTMs can be used in the forward mode to investigate the sensitivity of outputs, (i.e., surface reflectance and SIF) to different structural, biochemical, and physiological inputs, extending our mechanistic understanding of reflected and emitted photons' propagation across scales.

- Inverse mode. RTMs can be also inverted (i.e., run backwards) to estimate from laboratory, field and remote sensing spectral data those leaf and canopy traits that match measured reflectance and SIF data.

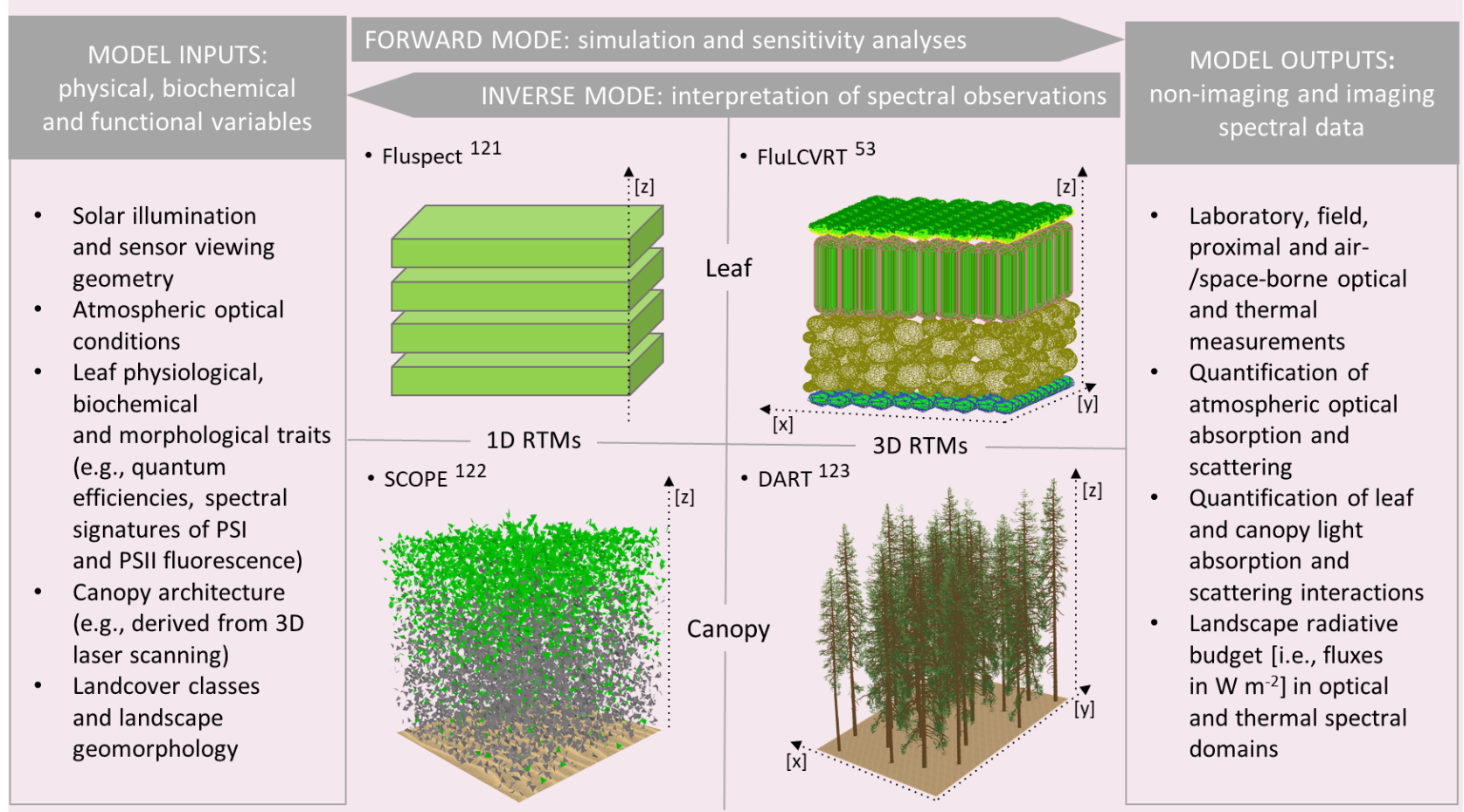

- 1D models. 1D leaf RTMs assume that leaf constituents are horizontally homogeneously distributed in vertically stacked plate structures, and hence require only basic morphological and biochemical inputs (e.g., pigment contents driving PAR absorption and within-leaf reabsorption, the intrinsic PSII and PSI fluorescence spectra, and the dynamics in the quantum yield of fluorescence as the mechanistic link to photosynthesis). This simplicity, however, ignores potentially important factors, such as within-leaf heterogeneity or chloroplast movements. As with the 1D leaf construct, 1D canopy RTMs assume that vegetation can be represented by horizontally homogeneous layers filled with leaves of a predefined size, density and geometry (angular distribution), which allows for minimal model inputs and a relatively straightforward application. The 1D architecture has its uses for spatially homogeneous canopies (e.g., crops).

- 3D models. Structurally complex leaves and spatially heterogeneous plant communities (e.g., forests and savannas) require 3D representations. 3D leaf RTMs can model optical interactions within a genuine 3D digital representation of leaf interior reconstructed, for example, with imaging tomography or confocal microscopy. As demonstrated in the Supplementary Videos 1 and 2, 3D RTM solutions also exist for spatially diversified plant canopies, allowing for accurate physical simulations of $\mathrm{APAR}_{\mathrm{g}}$ and $\mathrm{SIF}$ fluxes in complex canopies. 


\section{EXCITATION}

Solar spectral irradiance [W m-2 $\mathrm{nm}^{-1}$ ]

\section{PHOTOSYNTHETIC ENERGY PARTITIONING}

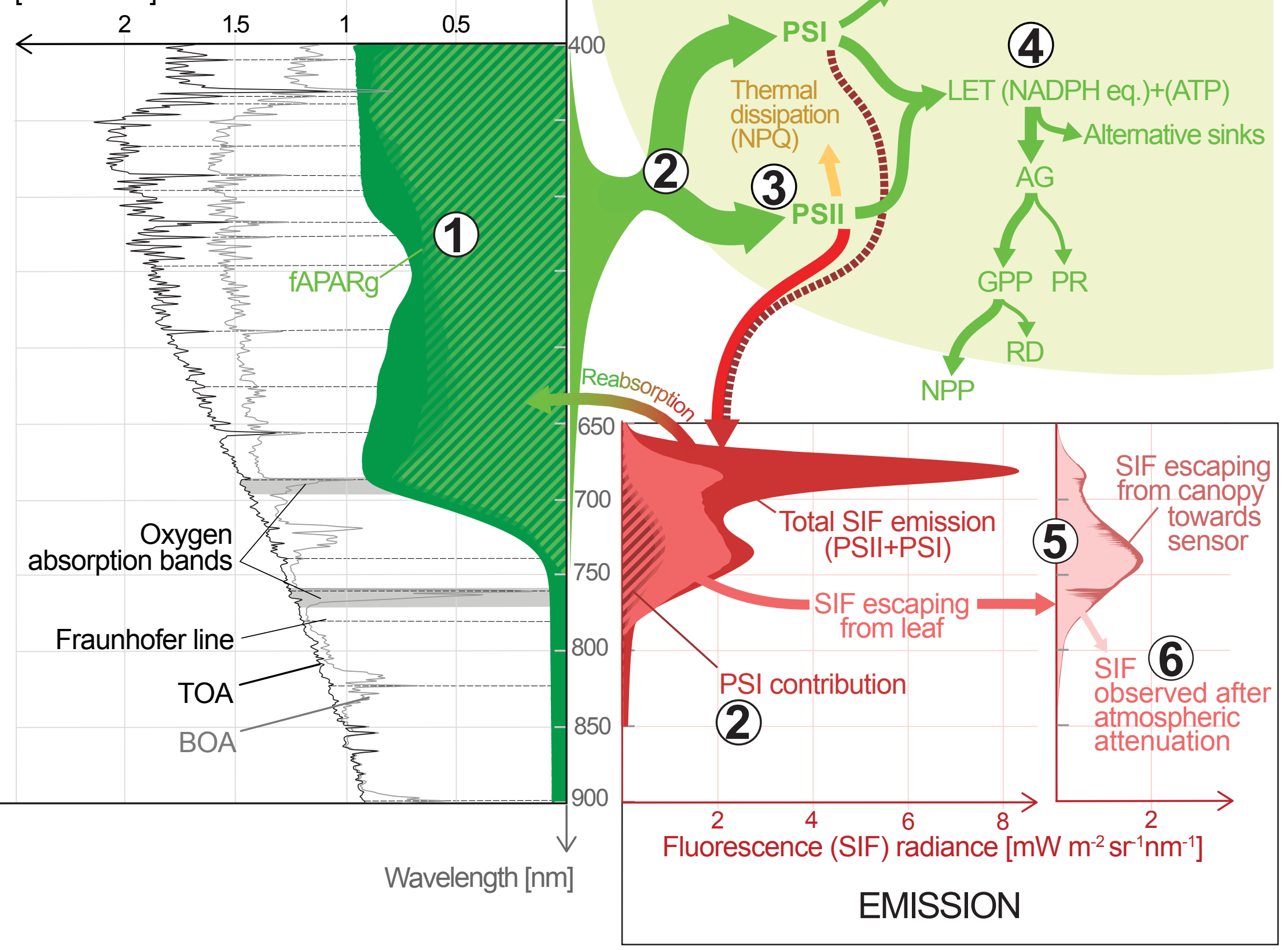




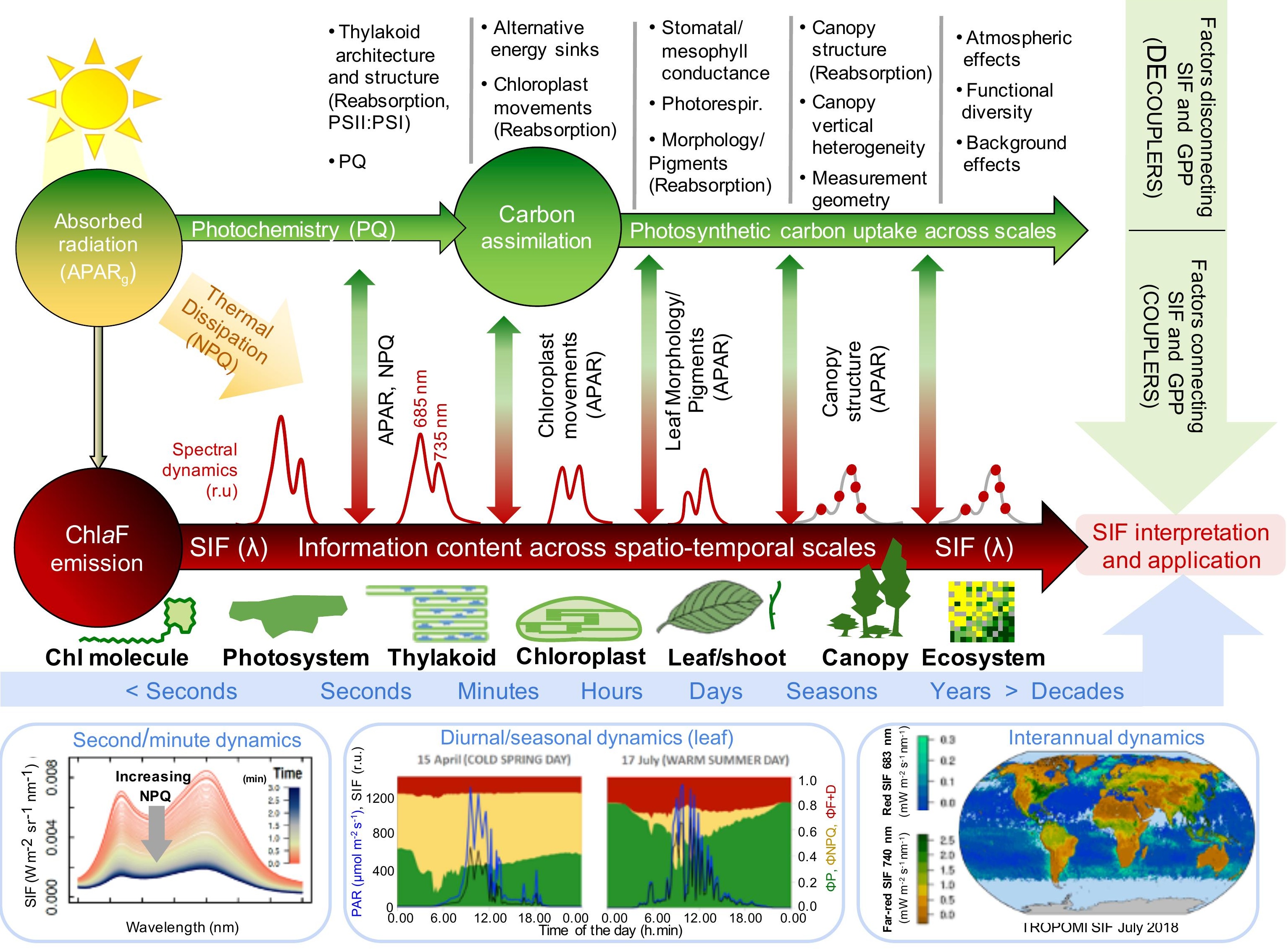




\section{CRITICAL STEPS/FACTORS}

INSTRUMENTAL

\section{(7) Standardized}

- measurement and calibration protocols

\section{ATMOSPHERIC}

(7)

Atm. corrections of

reflectance and SIF

observations (including

towers and drones)

\section{STRUCTURAL}

\section{(1) Improved parameterization}

5) of leaf and canopy RTMs

(7) with morphological and functional data

Standardization of canopy SIF in terms of efficiency

\section{PHYSIOLOGICAL}

Synergistic use of complementary/ancillary data

Full characterization of dynamics in photosynthetic energy partitioning between SIF and GPP

Comprehensive campaigns: long-

- term, multiple species, stressors and biomes
DATA SOURCES

METHODS

Instrument

characterization

and calibration data

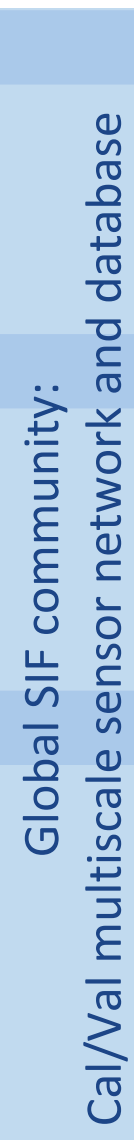
terrestrial and airborne LiDAR)

3D leaf morphology

Leaf and canopy empirical data for model calibration/validation (including PSI and PSII ChlaF spectra)

Leaf and plant empirical data for model calibration/validation:

- pigment content and dynamics

- PSI and PSII ChlaF contributions - energy partitioning between SIF and GPP

Data from a wide range of organisms (functional diversity)

Irradiance vertical profiles

Instrument calibrations

SIF retrieval methods

AT-SENSOR SIF/ REFLECTANCE

Atmospheric radiative transfer models (RTMs)

SPECTRAL OBSERVATIONS SIF/REFLECTANCE

Canopy \& leaf RTMs Spectral invariant - probability-based reflectance corrections

Process-based model development

Empirical models

Versatile protocols

to resolve PSI contribution and full energy partitioning

Multisource data

assimilation frameworks

\section{STRUCTURE}

CORRECTED

SIF/REFLECTANCE

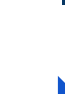

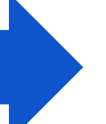

ECOSYSTEM,

CANOPY AND

LEAF

PHOTOSYNTHETIC TRAITS 


\section{Biochemistry \\ \& Biophysics}

Molecular regulation of photosynthesis

Experimental modeling

0

\section{Plant}

\section{Ecophysiology}

Functional dynamics in extreme \& remote biomes

Phenology \& circadian controls

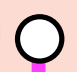

\section{(}

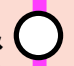

Functional \& spatial diversity

\section{Ecology}

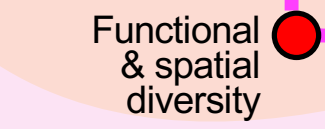

O

Investigating drivers of photosynthesis 3D photosynthesis: Plant structure \& function

Detecting plant

functional traits

Phenotyping

$\&$ stress

detection

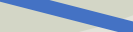

Breeding \&

\section{Agriculture \& Forestry}

Resource-use Optimized management

Pre-visual pest \& disease detection

$$
\text { new varieties }
$$
Precision egriculture \& forestry
Land cover/use change detection

Multiscale carbon \& water cycles

Biogeochemistry

Biosphere-atmosphere feedbacks

Impacts of climate change \& extreme events 